The University of Southern Mississippi

The Aquila Digital Community

Faculty Publications

$5-1-1993$

\title{
Evidence of a Nondiffusive Transport in a Monodisperse Screened Coulomb System by a Molecular-Dynamics Simulation
}

Michael Wild

University of Southern Mississippi

Ras B. Pandey

University of Southern Mississippi, ras.pandey@usm.edu

Follow this and additional works at: https://aquila.usm.edu/fac_pubs

Part of the Physics Commons

\section{Recommended Citation}

Wild, M., Pandey, R. B. (1993). Evidence of a Nondiffusive Transport in a Monodisperse Screened Coulomb System by a Molecular-Dynamics Simulation. Physical Review E, 47(5), 3246-3250.

Available at: https://aquila.usm.edu/fac_pubs/6479

This Article is brought to you for free and open access by The Aquila Digital Community. It has been accepted for inclusion in Faculty Publications by an authorized administrator of The Aquila Digital Community. For more information, please contact Joshua.Cromwell@usm.edu. 


\title{
Evidence of a nondiffusive transport in a monodisperse screened Coulomb system by a molecular-dynamics simulation
}

\author{
Michael Wild \\ Program in Scientific Computing, University of Southern Mississippi, Hattiesburg, Mississippi 39406-5046 \\ Ras B. Pandey \\ Program in Scientific Computing and Department of Physics and Astronomy, University of Southern Mississippi, \\ Hattiesburg, Mississippi 39406-5046
}

(Received 21 September 1992; revised manuscript received 3 February 1993)

\begin{abstract}
A molecular-dynamics simulation is carried out for particles (tracers) interacting with a screened Coulomb potential in three dimensions. A phase transition is observed from a liquid to an fcc solid on reducing the temperature at a fixed density, consistent with previous studies. In the liquid phase, the variation of the rms displacement $R_{\mathrm{tr}}$ of the charged particles with time seems to depend on the density and the temperature. In the short-time regime, the effective exponent $k$ for the subdiffusive power-law behavior, i.e., $R_{\mathrm{tr}} \sim t^{k}$, decreases on increasing the density and lowering the temperature. A crossover from subdiffusive to a diffusive behavior is observed for a wide density regime in the liquid phase. At high temperatures and large densities, a single power law does not seem to describe the variation of $R_{\text {tr }}$ with $t$.
\end{abstract}

PACS number(s): 61.20.Ja, 05.40. $+\mathrm{j}, 66.10 . \mathrm{Cb}$

The global transport phenomena on a mesoscopic scale, such as heat transfer, charge transport, and sound propagation in a fluid or solid depend on the dynamics at the microscopic scale of its constituent particles. Studying the tracers diffusion [1] in interacting model systems $[2-6]$ by computer simulations has attracted a considerable interest due to (i) enormous applicabilities [7] in monodisperse fluids and complex fluid mixtures, and (ii) intractability of the analytical methods [1] to take into account the nonlinear effects in highly correlated systems. Monodisperse systems with long-range interactions such as a Coulomb one and its variants are widely studied by Monte Carlo (MC) [8] as well as molecular-dynamics (MD) [9] methods. Most of these investigations deal with the analysis of structural properties such as liquid to crystalline transition [10-14], glass formation [15], and the structural instabilities [16] associated with various phases as the system evolves on changing the parameters such as the temperature, density, range of interaction, etc. However, comparatively few attempts are made to understand the diffusive process of the particles in such evolutionary systems. Very recently, Ould-Kaddour and Barrat [6] have reported a (MD) study of tracers' diffusion with a truncated Lennard-Jones potential where deviations from a Stokes-Einstein behavior of the diffusion coefficient was discussed; in their study, the variation of the root-mean-square (rms) displacement of tracers with time was taken to be a Fickian diffusion. Using a MC simulation, Maass et al. [4], have observed a non-Debye relaxation of tracers with Coulomb interaction, though in a quenched inhomogeneous (percolating) medium; the power-law behavior of the rms displacement of even a noninteracting lattice gas is anomalous in such random systems. A subdiffusive power-law behavior of tracers in a nearest-neighbor-interacting lattice gas (without the quenched impurities) has also been reported
[16] recently. Here we present a MD simulation of a monodisperse system with a screened Coulomb (i.e., Yukawa) potential, in which we find a subdiffusive behavior of the rms displacement $\left(R_{\mathrm{tr}}\right)$ of the tracers in the liquid phase which is consistent with the recent observation of an intermediate subdiffusive regime by Robbins et al. [11]. This subdiffusive transport is described by an effective power-law exponent $k, R_{\mathrm{tr}} \sim t^{k}$, where $k$ depends on the temperature and density. In addition, we find an unusual transport behavior which cannot be described by a single power law.

We consider the Yukawa potential between two particles of the form,

$$
U(r)=(\varepsilon / r) \exp (-r / \xi),
$$

where $\varepsilon=q^{2} /\left(4 \pi \epsilon_{0}\right)$ with charge $q$ on each particle, $r$ is the linear separation between the particles, and $\xi$ is the screening length; both lengths are measured in units of $\sigma$ which depends on the specific system, but it is an arbitrary scaling parameter for our study. The force between the two particles can then be obtained from the spatial derivative of the potential. For example, the $x$ component of the force,

$$
\begin{aligned}
F_{x}=-\nabla_{x} U(r)=\left(x \varepsilon / \sigma^{3}\right)[ & (1 / A)(\sigma / R)^{2} \\
& \left.+(1 / R)^{3}\right] \exp (-R / A),
\end{aligned}
$$

where $R \sigma=r$ and $A \sigma=\xi$. Thus the separation distance and screening length is measured in units of $\sigma$ and the time in units of $\tau=(m \sigma / \varepsilon)^{1 / 2}$, as is usually done in MD simulations [9]. The flexibility in variation of the range of effective interaction from hard core $(\xi \rightarrow 0)$ to the long-range Coulomb $(\xi \rightarrow \infty)$ has been well emphasized in the literature [11-14]. We will restrict ourselves here only to a certain range (see below). Most of our data 
were obtained with 864 particles at several temperatures for a variety of densities, although we have used different numbers of particles (at a fixed density) to check the reliability of our results. The standard Verlet algorithm [9] is used with a periodic boundary condition and a normalized time step of 0.032 . We have used four thousand time steps to equilibrate the samples; during this initial period, the velocities are scaled every fifty steps to reach the equilibrium efficiently. Note that the velocities are not rescaled thereafter as the system remains at a constant energy in its equilibrium - a microcanonical ensemble system. Four to five thousand additional steps are used in most runs to analyze the equilibrium quantities; we have occasionally used as large as $3 \times 10^{4} \mathrm{MD}$ steps. Different initial configurations such as random, fcc, and simple cubic were used and their initial velocities were assigned with Boltzmann distribution. Physical quantities such as rms displacement of the particles, paircorrelation functions, velocity autocorrelation function, and energy were calculated as a function of scaled density, temperature, and the screening length.

We have explored both liquid and solid phases at a wide range of temperatures; however, we will focus here on the interesting transport regime in liquid phase. Figure 1(a) shows a typical evolution of the total energy with time at several scaled temperatures $T=0.001,0.002, \ldots$, 0.005 with the screening length $\xi=0.50$ starting with an fcc structure of the initial distribution of the particles; the temperature is scaled with the kinetic energy where the scaled velocity $v=1.13(2 T)^{1 / 2}$. Figure $1(\mathrm{~b})$ shows the variation at the temperature $T=0.002$ for densities $\rho=0.05,0.15$, and 0.20 . We see that the system reaches its equilibrium configuration rather well within a thousand MD times steps. The energy gap between consecutive temperatures seems to indicate a discontinuity as we increase the temperature from 0.002 to 0.003 [Fig. 1(a)]. We believe that the two lower curves at the lower temperatures give the equilibrium energy associated with the solid phase while the higher energies correspond to liquid phase at temperature larger than 0.002 at the fixed density 0.40 . Thus, at density 0.40 , the melting temperature of our model falls between 0.002 and 0.003 , where the discontinuity in the energy gap is a measure of the energy associated with the latent heat.

Figure 2 shows the variation of the pair-correlation function $g(r)$ with the interparticles distance $r$ for the same parameters as in Fig. 1(a). At the low temperature (0.001), the pronounced peaks are formed at well-defined positions which are roughly the distance for the successive neighbors of a fcc lattice. These peaks become diffused at the higher temperature (0.002). On further increasing the temperature above 0.002 , we observe a dramatic change in the variation of $g(r)$ with $r$. The first pronounced peak, which corresponds to a short-range order, is followed by damped peaks which show extremely weak order beyond the first neighbor and lack of longrange order. All the data points at higher $T$ fall almost on the same curve, except for the first pronounced peak. Such variation of the correlation function is typical of the liquid phase. Thus our observation of liquid to fec solid transition is consistent with recent $\mathrm{MC}[12,13]$ as well as
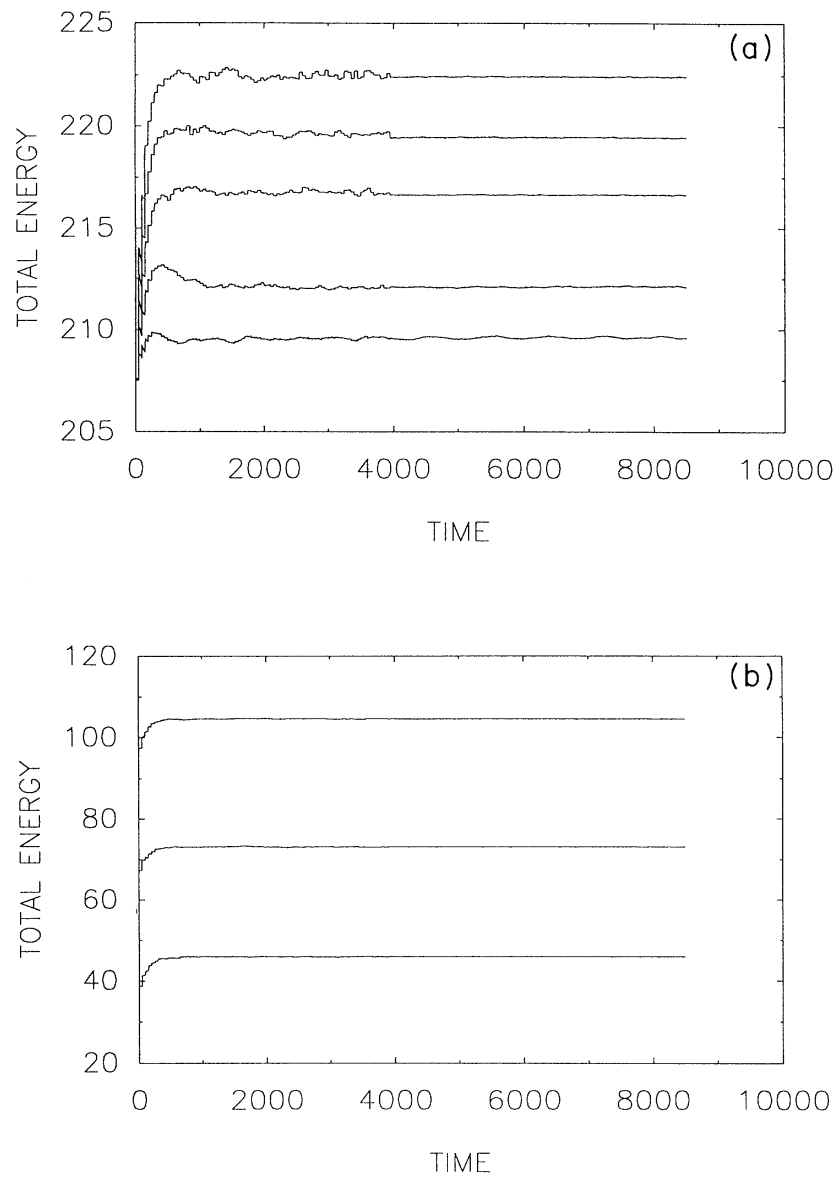

FIG. 1. Total energy vs time with the screening-length parameter $\xi=0.50$, and cutoff range $r=3.5$ in this and the following figures. (a) At a fixed density 0.40 with 864 particles for various temperatures beginning at 0.001 from bottom curve with an increment of 0.001 . (b) At a fixed temperature $T=0.002$ for different densities from bottom to top $\rho=0.15$, 0.20 , and 0.20 . fcc lattice for the initial configuration of the particle distribution is used in Figs. 1-6.

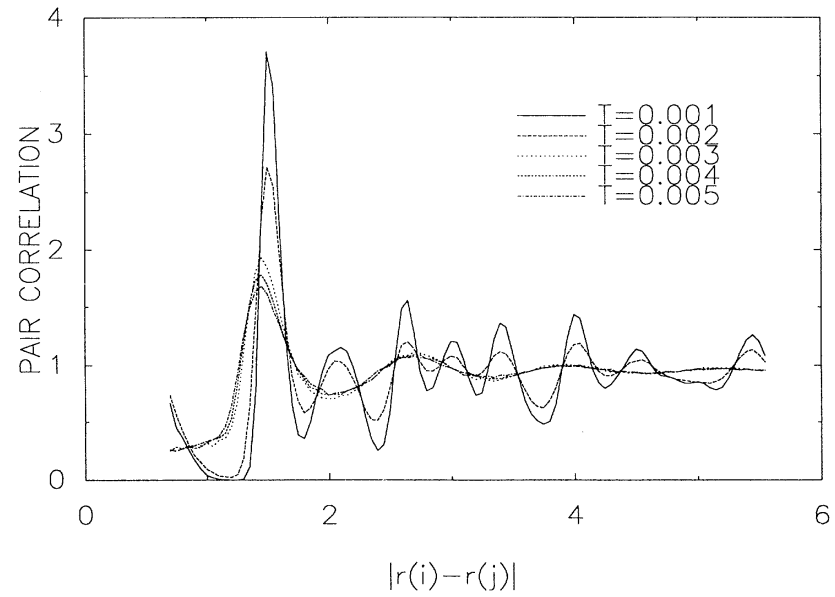

FIG. 2. Pair-correlation function vs separation distance for the same parameters and statistics as in Fig. 1(a). 
MD [11,14] simulations.

The variation of the rms displacement of the particles, as the system undergoes a phase transition from liquid to an fcc solid phase, will now be examined as the temperature is reduced. The power-law dependence of the rms displacement $R_{\mathrm{tr}}$ with time $t$ is usually described by $R_{\mathrm{tr}} \sim t^{k}$, where the exponent $k=\frac{1}{2}$ for the Fickian diffusion and $k<\frac{1}{2}$ for anomalous diffusion [1,17] as well as for the subdiffusive behavior [16]. Figure 3 is a plot of
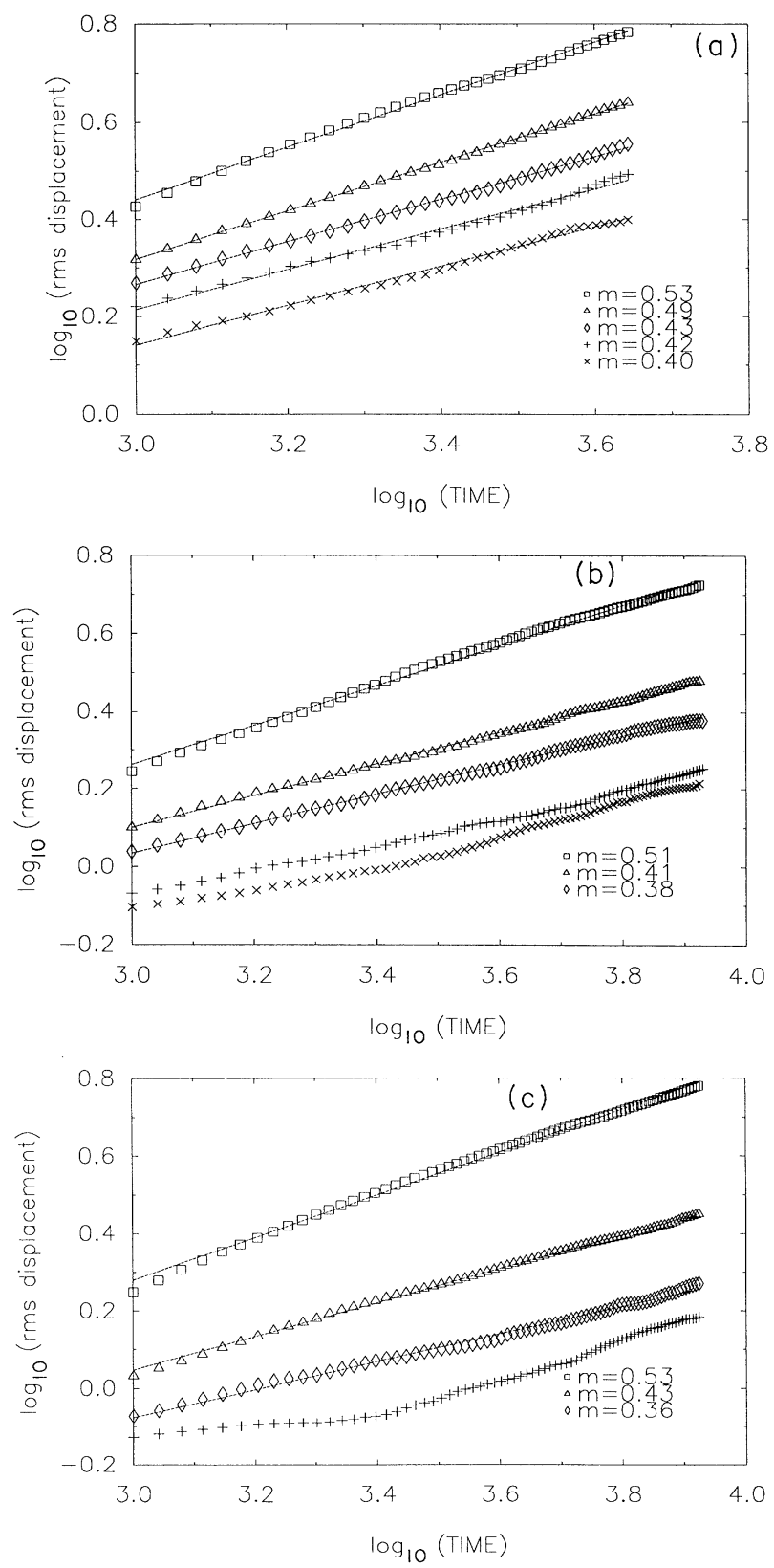

FIG. 3. Log-log plot of the rms displacement vs time at temperatures: (a) $T=0.007$ for densities $\rho=0.10,0.20,0.30,0.40$, 0.50 , (b) $T=0.003$ for densities $\rho=0.10,0.20,0.30,0.40,0.42$, and (c) $T=0.002$ for densities $\rho=0.05,0.15,0.20,0.25$ from the top to bottom. The inset numbers are the slopes of the leastsquares fit (broken lines) of those data which show power-law dependence from top to bottom. the rms displacement versus time for various densities. We observe a diffusive behavior at low density, $k=0.52 \pm 01$ at $\rho=0.10, T=0.007$ [Fig. 3(a)]. On increasing the density, we observe a continuous deviation from diffusion to a subdiffusive power-law behavior where the effective exponent $k$ decreases accordingly. This subdiffusive behavior becomes more pronounced as $T$ is lowered even at lower densities [Figs. 3(b) and 3(c)]. Figure 4 sows a systematic decrease in the slope of $\log _{10}\left(R_{\mathrm{tr}}\right)$ vs $\log _{10}(t)$ (i.e., $k$ ) on reducing the temperature from 0.007 to 0.003 at a fixed density $\rho=0.40$. It is worth mentioning here that the difference in diffusive and subdiffusive transport behavior is also evident from the variation of the velocity autocorrelation function with time (see Fig. 5).

We would like to point out that we have done our stability analysis with different time steps. We find that the approach of energy to its equilibrium value remains qualitatively the same with different time steps (i.e., 0.064, 0.032 , and 0.010 ), and we have used the time step 0.032 for all our data as we have mentioned in the beginning. Evaluation of the effective exponent $k\left(R_{\mathrm{tr}} \sim t^{k}\right)$ to a good accuracy is difficult due to large error bars. One may, however, look at the variation in the slope of the consecutive data points in $\log _{10}\left(R_{\mathrm{tr}}\right)$ vs $\log _{10}(t)$ plot. Figure 6 shows a typical plot of $k$ vs $t$ at $\rho=0.40$ and $T=0.004$ for the time steps 0.032 and 0.010 , respectively. The large fluctuations in $k$ here only describe the local stochastic hopping and not the global power laws as we discussed in the preceding paragraph. Note that reducing the time step results in larger fluctuations in $k$ which reveals the details of stochastic movement. If we probe the microscopic details of the particles' movements on smaller scales by reducing the time steps, we may see more noisy data (closer to realistic systems) which is not the focus of this study. However, one should not be confused with the fluctuations in $k$ of the consecutive data points here and the power-law exponents $k$ of the preceding sections (i.e., Figs. 4 and 5) which describe the overall transport behavior.

One may argue that the physical mechanism underly-

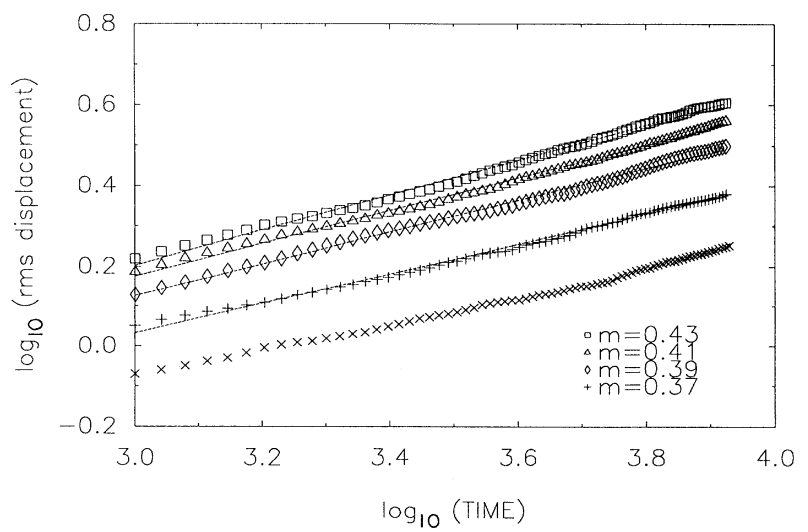

FIG. 4. Log-log plot of the rms displacement vs time at a fixed density $\rho=0.40$, at temperatures $T=0.003-0.007$ at an increment of 0.001 from the bottom with the same statistics as in Fig. 3. The broken lines are the linear fit. 


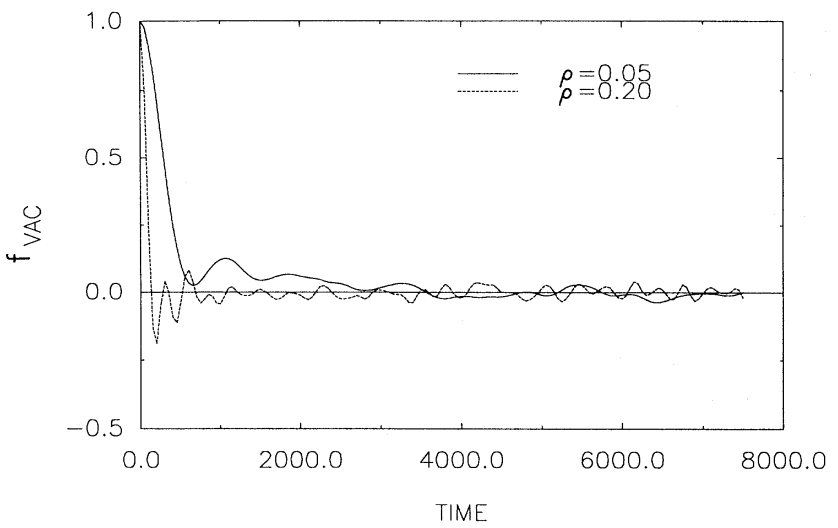

FIG. 5. Velocity autocorrelation function $f_{\text {vac }}$ vs time at temperature $T=0.002$ for densities $\rho=0.05$ (solid line) and 0.20 (broken line) for the same statistics as that of Fig. 3.

ing the subdiffusive motion is the nature of the effective medium in which each particle moves. Distribution of particles within the range of interaction (the screening length) determines the local medium which may change as the particles move. It is likely that each particle, on average, moves in an inhomogeneous medium, at least in a certain time regime, which depends on the density and temperature. The motion of a single particle in an inho-
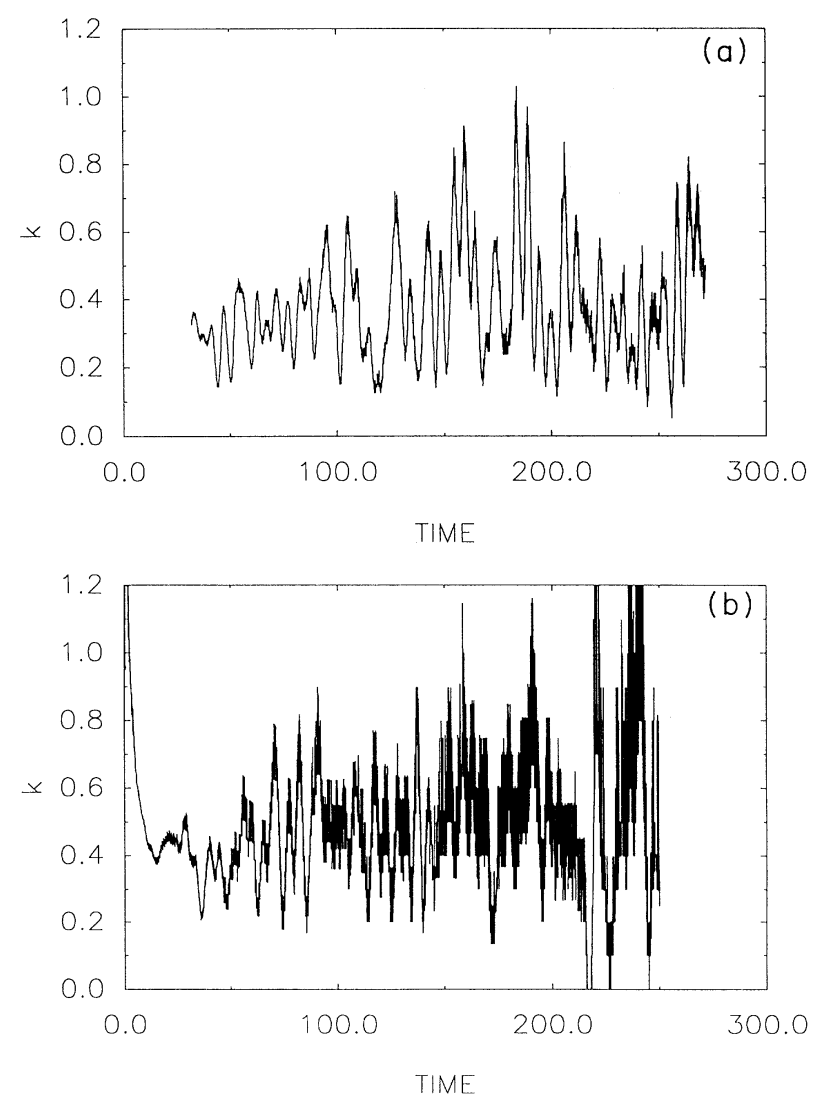

FIG. 6. Variation of the effective exponent $k$ with time $t$ at density $\rho=0.40$ and temperature $T=0.004$ with time steps (a) 0.032 and (b) 0.010 . mogeneous media may lead to a subdiffusive transport behavior. The range of this subdiffusive regime increases on increasing the density and lowering the temperature. Beyond this regime, an asymptotic diffusive behavior is expected as the particles move effectively in a homogeneous media on a larger scale. Within the range of our observation time, we have not noticed such a crossover to a diffusive behavior at low temperatures with a fcc initial condition but with a random initial condition (see below). This suggests that the effective medium for the particles' subdiffusive transport is perhaps inhomogeneous. If one associates a correlation length (as the percolation correlation length [17]) with the distribution of the potential barriers, then this length is at least as large as the rms displacement of the particles.

Similar crossover from diffusive to subdiffusive transport behavior is also observed with random initial configuration as the density is increased (Fig. 7). At high temperature, $T=0.007$, we also observe a crossover from a subdiffusive behavior at short time to a diffusive behavior in long time (Fig. 8). According to our effective medium picture above, the range of inhomogeneity is relatively shorter in comparison with the fcc initial configuration. Such crossover in time is already observed in other interacting lattice-gas simulations [16]. Excellent linear fit of these data suggests that a subdiffusive power-law behavior exists at least in a certain time regime (within our observation time range). Thus we see that a monodisperse Yukawa liquid exhibits a subdiffusive power-law behavior for tracers' transport in certain density regimes, like the Coulomb particles in inhomogeneous matrix [4], or the binary mixtures of $\mathrm{Yu}$ kawa particles [5]. We should point out that in literature $[6,11]$, tracer transport is treated as diffusive by scaling the rms displacements by $t^{2}$ in order to calculate the diffusion constant; such treatments do not apply here in subdiffusive $\rho-T$ regime as the prefactor of our power-law relation does not represent the Fickian diffusion constant. Simulations with the random initialization show an unusual variation of the rms displacement at high temperature $(T=0.007)$ and high density $(\rho=0.50,0.70)$; the variation of some of these data cannot be explained

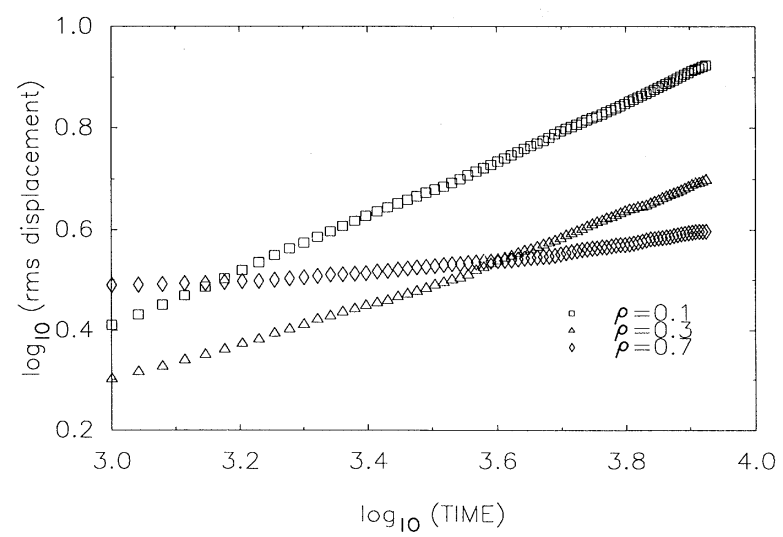

FIG. 7. With the random initial configuration, the log-log plot of the rms displacement vs time at a fixed temperature $T=0.007$, for various densities $\rho=0.10,0.30,0.50$, and 0.70 . 


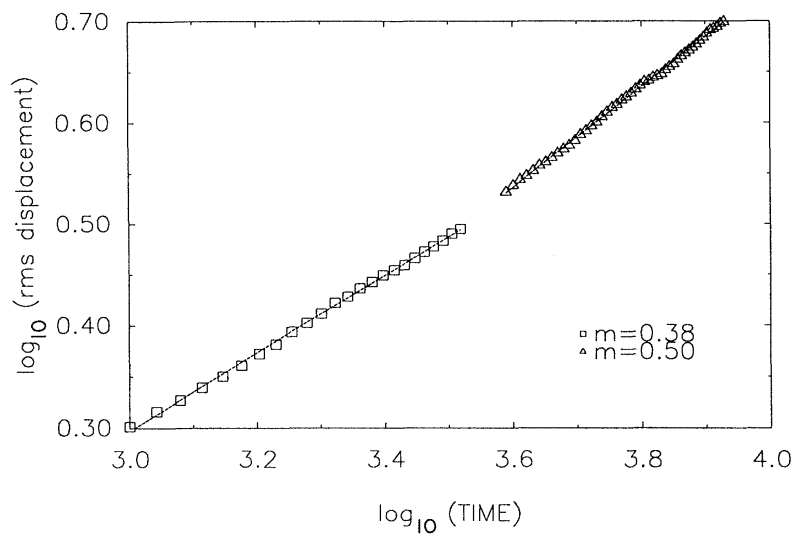

FIG. 8. Log-log plot of rms displacement of a typical variation of the rms displacement vs time at the temperature $T=0.007$ and the density $\rho=0.30$ to show the crossover from a subdiffusive to a diffusive transport behavior with the same statistics as that of Fig. 6 .

by a single power-law exponent discussed so far. We would like to point out that, in contrast to liquid-solid transition with fcc initial configurations, we never observed a crystalline solid phase on varying the density at a fixed temperature with random initial configurations.
The system seems to remain in liquid state even at very high densities where the rms displacement increases extremley slowly - an ultra-slow transport; such $\rho-T$ regime may be associated with a supercooled liquid state.

In conclusion, a MD simulation is presented to study a monodisperse Yukawa particles system. With an fcc initial configuration, we find that a monodisperse Yukawa undergoes a phase transition from liquid to a fcc solid on increasing the density and reducing the temperature. The rms displacement of tracers exhibits a subdiffusive power-law behavior near melting over the entire range of our observation time at low temperatures. This behavior is described by an effective power-law exponent $k$ which depends on the temperature and density. With the random initial configurations, on the other hand, we never observe an ordered solid phase. At low temperatures, we observe a crossover from a subdiffusive behavior in short-time regime to a diffusive behavior in long time. At high densities, the rms displacement cannot be described by a single power law. This behavior is similar to transport in quenched inhomogeneous systems [4] and the mixtures of charged particles [5].

We would like to thank G. S. Dubey, G. Grest, and K. Kremer for useful discussions. R.B.P. acknowledges a partial support from the NSF EPSCOR Grant No. EHR9108767.
[1] J. W. Haus and K. W. Kehr, Phys. Rep. 150, 263 (1987); S. Havlin and D. Ben-Avraham, Adv. Phys. 36, 695 (1987).

[2] R. B. Pandey, Phys. Rev. A 42, 3363 (1990).

[3] Y. Gefen and J. W. Hallye, in Kinetics of Aggregation and Gelation, edited by F. Family and D. P. Landau (NorthHolland, Amsterdam, 1984).

[4] P. Maass, J. Petersen, A. Bunde, W. Dietrich, and H. E. Roman, Phys. Rev. Lett. 66, 52 (1991); P. Maass, A. Bunde, and M. D. Ingram, ibid. 68, 3064 (1992); R. B. Pandey, Physica A 191, 438 (1992).

[5] K. Kremer, G. S. Grest, and M. O. Robbins, J. Phys. A 20, L181 (1987).

[6] F. Ould-Kaddour and J-L. Barrat, Phys. Rev. A 45, 2308 (1992).

[7] Physics of Complex and Supermolecular Fluids, edited by S. A. Safran and N. A. Clark (Wiley, New York, 1987).

[8] Application of Monte Carlo Method in Statistical Physics, edited by K. Binder (Springer-Verlag, Berlin, 1987).

[9] M. P. Allen and D. J. Tildesley, Computer Simulation of
Liquids (Clarendon, Oxford, 1989); D. W. Heermann, Computer Simulation Methods in Theoretical Physics (Springer-Verlag, Berlin, 1990).

[10] D. Levesque, J. J. Weis, and J. P. Hansen, in Application of Monte Carlo Method in Statistical Physics, edited by $\mathrm{K}$. Binder (Ref. [8]), p. 37.

[11] M. O. Robbins, K. Kremer, and G. S. Grest, J. Chem. Phys. 88, 3286 (1988).

[12] E. J. Meijer and D. Frenkel, J. Chem. Phys. 94, 2269 (1991).

[13] B. B. Laird and A. D. J. Haymet, Mol. Phys. 75, 71 (1992).

[14] R. O. Rosenberg and D. Thirumalai, Phys. Rev. A 33, 4473 (1986); 36, 5690 (1987).

[15] S. Rangnathan and K. N. Pathak, Phys. Rev. A 45, 5789 (1992); S. Ranganathan, G. S. Dubey, and K. N. Pathak, ibid. 45, 5793 (1992).

[16] R. B. Pandey, Physica A 187, 77 (1992).

[17] D. Stauffer and A. Aharony, Introduction to Percolation Theory, 2nd ed. (Taylor and Francis, London, 1992). 